\title{
A participação social na vigilância de medicamentos
}

Social participation in drug surveillance

La participación social en la vigilancia de los medicamentos

\author{
Julino Rodrigues Soares ${ }^{1}$ \\ Vanessa Elias de Oliveira ${ }^{2}$
}

\section{Resumo}

Objetivo: analisar a contribuição dos pacientes na vigilância de medicamentos, considerando algumas etapas de desenvolvimento dessas tecnologias e o interesse da saúde pública. Metodologia: foi realizada análise bibliográfica e documental sobre as contribuições dos pacientes e os mecanismos de participação social. Resultados: identificou-se iniciativas consolidadas e resultados que sustentam a importância do paciente na vigilância de medicamentos em diversos segmentos. Todavia, a participação é limitada devido ao baixo letramento científico e à inefetividade de alguns mecanismos de participação social. Conclusões: organizações representativas de pacientes podem contribuir mais efetivamente em iniciativas de participação social na vigilância de medicamentos, conforme relatado em experiências internacionais. Também, é fundamental que as agências reguladoras busquem aprimorar os mecanismos de participação e a utilização de métodos que possibilitem coletar as contribuições sociais, bem como a sistematização das experiências dos pacientes.

Palavras-chave: Vigilância sanitária. Farmacovigilância. Participação do paciente. Políticas públicas de saúde.

\begin{abstract}
Objective: to analyze the contribution of patients in drug surveillance, considering some stages of development of these technologies and the interest of public health. Methodology: a bibliographic and documentary analysis was performed on patients' contributions and mechanisms of social participation. Results: we identified consolidated initiatives and results that support the importance of patients in drug surveillance in various segments. However, participation is limited due to the low scientific literacy and ineffectiveness of some social participation mechanisms. Conclusion: patient representative organizations can contribute more effectively to social participation initiatives in drug surveillance, as reported in international experiences. In addition, it is essential that regulatory agencies seek to improve participation mechanisms and the use of methods to collect social contributions, as well as to systematize patients' experiences.
\end{abstract}

Keywords: Sanitary surveillance. Pharmacovigilance. Patient participation. Public health policies.

\footnotetext{
1 Doutor em Psicobiologia pela Universidade Federal de São Paulo, São Paulo, Brasil; pós-doutorando na Universidade Federal do ABC, São Paulo, Brasil. http://orcid.org/0000-0002-3995-8908. E-mail: julino.rodrigues@gmail.com

${ }^{2}$ Cientista Política pela USP, Professora Associada e Coordenadora da Pós-Graduação em Políticas Públicas da UFABC. E-mail: vanessa.oliveira@ufabc.edu.br
} 


\section{Resumen}

Objetivo: analizar la contribución de los pacientes a la vigilancia de medicamentos, considerando algunas etapas del desarrollo de estas tecnologías y el interés de la salud pública. Metodología: se realizó un análisis bibliográfico y documental sobre las contribuciones de los pacientes y los mecanismos de participación social. Resultados: identificamos iniciativas y resultados consolidados que respaldan la importancia de los pacientes en la vigilancia de medicamentos en diversos segmentos. Sin embargo, la participación es limitada debido a la baja alfabetización científica y la ineficacia de algunos mecanismos de participación social. Conclusion: Las organizaciones representativas de pacientes pueden contribuir más eficazmente a las iniciativas de participación social en la vigilancia de drogas, como se informa en experiencias internacionales. Además, es esencial que las agencias reguladoras busquen mejorar los mecanismos de participación y el uso de métodos para recaudar contribuciones sociales, así como para sistematizar las experiencias de los pacientes.

Palabras-Ilave: Vigilancia sanitaria. Farmacovigilancia. Participación del paciente. Políticas públicas de salud.

\section{Introdução}

A Constituição Federal Brasileira de 1988 estabelece, em seu artigo 198, inciso III, a participação da comunidade como um dos pilares do Sistema Único de Saúde (SUS). Os órgãos e entidades públicas de saúde têm como missão a incorporação ampla e efetiva de mecanismos de participação social, disponibilizando espaços e instrumentos para parcerias regulatórias em sistemas complexos $(1,2)$.

Apesar da institucionalização de determinados espaços de participação social, como os conselhos de saúde e as conferências de saúde, estes não são suficientes para garantir a efetiva participação e a influência dos atores sociais nos processos de tomada de decisão e elaboração de políticas públicas. Isso porque, conforme diversos estudos demonstram, a adequada participação social no Conselho de Saúde encontra dificuldades, sobretudo, em razão de conflitos de interesse, assimetrias sociais e de conhecimento $(3,4)$.

O insuficiente envolvimento social em saúde impacta diversas áreas desta política pública, como os sistemas de vigilância sanitária, que enfrentam desafios no cumprimento de suas atividades. Alguns desses desafios merecem maior atenção e reflexão por parte da sociedade, visto que falhas nas atividades de vigilância sanitária de medicamentos impactam diretamente na saúde dos pacientes e na prática clínica dos profissionais da saúde. O aprimoramento da participação social poderia contribuir significativamente para a vigilância de medicamentos e, portanto, para a segurança dos pacientes. 
Nos últimos anos a Agência Nacional de Vigilância Sanitária (Anvisa) vem enfrentando dificuldades para manter algumas decisões perante o legislativo, abrindo questionamentos sobre a qualidade regulatória no Brasil. Podemos citar como exemplo o Decreto Legislativo $n^{\circ}$ 273/2014, aprovado pelo Congresso Nacional, que invalidou a Resolução da Diretoria Colegiada (RDC no 52/2011) que proibia o uso dos inibidores do apetite anfepramona, femproporex e mazindol, e impunha medidas de controle da prescrição e dispensação da sibutramina (5), o que os obrigou a aprovar um novo regulamento para o uso de anorexígenos (RDC no 50/2014) (6).

Outro exemplo que pode impactar na vida dos pacientes e dos profissionais da saúde é a qualidade das bulas. Mesmo após a publicação da Resolução da Diretoria Colegiada (RDC no 47/2009) pela Anvisa, observou-se que algumas das bulas analisadas apresentaram informações incompletas ou divergentes das descritas nas fontes consultadas (Dicionário Terapêutico Guanabara e Micromedex Database) (7) e ainda possuíam uma linguagem de difícil compreensão para os pacientes, letras pequenas, ausência de ilustrações ou uso incorreto das mesmas nas bulas (8).

A agência norte-americana de medicamento, Food and Drug Administration (FDA), também foi criticada pela qualidade das suas aprovações de medicamentos e sistema de pós-comercialização no caso dos inibidores da COX-2, além de sofrer questionamentos sobre conflito de interesses. Esse fato desencadeou uma pesquisa com quase 400 cientistas da FDA, que se tornou pública através de um pedido de liberdade de informação. A pesquisa indica que quase um quinto dos cientistas foi pressionado a aprovar um novo medicamento apesar das ressalvas quanto à sua segurança. A maioria dos cientistas entrevistados também disse que duvida da capacidade da FDA de monitorar medicamentos prescritos na pós-comercialização (9).

Devemos considerar a complexidade de todo esse cenário e os desafios para garantir a segurança dos pacientes e, nesse processo multifacetado, a participação social pode contribuir com maior transparência e o aprimoramento da vigilância de medicamentos. Algumas iniciativas internacionais indicam a possibilidade de inovação na participação social em saúde, especialmente na participação de pacientes na vigilância de medicamentos (10, 11). Dessa forma, é imperioso considerar a aplicabilidade desses mecanismos de participação social no Brasil e como o diálogo por eles proporcionado poderia contribuir para 
a melhor regulação de substâncias medicinais e, por conseguinte, da saúde dos pacientes. Assim, o presente estudo visa analisar a contribuição dos pacientes na vigilância de medicamentos, considerando algumas etapas de desenvolvimento dessas tecnologias e o interesse da saúde pública.

\section{Método}

Foi realizada análise bibliográfica e documental sobre a participação social em atividades de vigilância sanitária com ênfase em farmacovigilância. Para isso, foi realizado um levantamento de dados sobre os mecanismos de participação social diretamente no site da European Medicines Agency (EMA), U.S. Food and Drug Administration (FDA), e da Agência Nacional de Vigilância Sanitária (ANVISA).

A literatura utilizada para a fundamentação teórica e discussão dos achados foi levantada na base de dados digital PubMed da US National Library of Medicine National Institutes of Health e no Google Acadêmico, além de livros de referência.

Foram considerados os descritores e qualificadores autorizados (DECs): patient participation, pharmacovigilance, health surveillance, consumer organizations, health public policies, health education.

\section{O engajamento do paciente na vigilância de medicamentos}

O lançamento de um medicamento no mercado é parte, e não o fim, do seu processo de desenvolvimento. Contudo, os diminutos investimentos existentes na póscomercialização, em relação às etapas de pesquisa clínica, dificultam a avaliação do perfil de segurança e efetividade. De todo modo, as ações de vigilância sanitária perduram enquanto o medicamento estiver sendo comercializado (12).

Nos EUA, estima-se que a demanda por prazos menores para a aprovação de medicamentos (The Prescription Drug User Fee Act) possa resultar em problemas na segurança dos medicamentos. As aprovações da FDA antes do prazo, em relação às aprovadas no prazo regular, resultaram na retirada do mercado de aproximadamente $13 \%$ desses medicamentos. Além disso, $10 \%$ dos medicamentos receberam tarjas pretas e $3 \%$ sofreram descontinuação ou modificações na dosagem após a aprovação (13). 
Quando o medicamento já está em uso pela população, uma das etapas fundamentais é a vigilância pós-comercialização, uma vez que os estudos pré-clínicos e clínicos não são suficientes para garantir a total segurança do paciente. A sociedade, em especial os pacientes e os seus familiares, pode trazer inegáveis contributos nas diversas etapas de avaliação dos medicamentos, notadamente quanto aos riscos, às pesquisas clínicas, aos aspectos éticos e também nas discussões sobre o acesso e adesão terapêutica, haja vista sua experiência de uso.

A notificação de eventos adversos a medicamentos (EAM) ainda é um dos métodos mais utilizados para detectar problemas relacionados a medicamentos quando esse já está em uso pela população. A notificação de EAM, seja compulsória ou espontânea, possibilita detectar rapidamente os erros de medicação, o uso de medicamentos para indicações não aprovadas (off-label), bem como casos de intoxicação, abuso e uso indevido, interações medicamentosas adversas, dentre outros (14).

Como existem muitas subnotificações de EAM ou mesmo notificações de baixa qualidade, os pacientes devem ser estimulados a fazerem notificações espontâneas de EAM, como uma importante forma de apoio à farmacovigilância. Segundo o relatório Direct Patient Reporting of Adverse Drug Reactions: a fifteen-country survey \& literature review, as contribuições dos pacientes na área da segurança de fármacos são fundamentais para a saúde pública (15). Como salientam os referidos autores, a notificação realizada diretamente por pacientes possibilita acelerar a aquisição de conhecimento sobre os medicamentos, pois, além de ser mais direta do que aquelas realizadas pelos profissionais da saúde, também acaba por incluir relatos sobre o impacto dos EAM na vida dos pacientes e familiares.

A participação do paciente na regulação e vigilância de medicamentos ainda é, de fato, um desafio. No entanto, já é possível verificar a realização de ações, nomeadamente no âmbito da EMA e da FDA, por exemplo, para incluir os pacientes e suas organizações representativas em diversas iniciativas.

Como um reflexo disso, desde 2006 a FDA recebeu mais notificações de pacientes do que aquelas provenientes de médicos e de farmacêuticos. As notificações de EAM enviadas por pacientes, que no ano 2000 totalizavam 46.249, chegaram a 272.989 em 2009 (16). 
Na Europa, o Comitê de Medicamentos Órfãos da EMA (COMP) incluiu a participação de pacientes desde o ano 2000 e, desde 2012, conta com a inclusão de um representante dos pacientes no Comitê de Farmacovigilância e Avaliação de Riscos (PRAC, em inglês). Essa abertura à participação tem como objetivo contribuir com a EMA na aquisição de conhecimentos sobre experiências da vida real sobre a doença; no tratamento e conhecimentos específicos para discussões científicas sobre medicamentos; bem como possibilitar uma comunicação mais efetiva com os pacientes, reforçar a compreensão do papel da rede de regulamentação dos medicamentos da União Europeia e do impacto das decisões regulatórias (10).

Os pacientes e consumidores podem se envolver em uma ampla gama de atividades na EMA como membros do conselho de administração e dos comitês científicos: participar de discussões sobre o desenvolvimento e autorização de medicamentos; fazer a revisão de informações preparadas pela EMA sobre medicamentos; estar envolvido na elaboração de diretrizes, dentre outros. Em 2017, a EMA realizou 925 atividades envolvendo pacientes e consumidores, dos quais 400 eram representantes de organizações sem fins lucrativos (10).

Nos Estados Unidos, a FDA incluiu a perspectiva do paciente nas reuniões do seu Comitê Consultivo desde 1991. Os pacientes e seu familiares podem se envolver em iniciativas que se relacionem com o desenvolvimento de medicamentos e com doenças raras, a partir de relatos de experiência de uso (11). Entretanto, vale ressaltar que para participar dos seus Comitês Consultivos, o representante dos pacientes deve ser capaz de analisar dados científicos, entender o desenho das pesquisas, discutir os riscos e benefícios, e ser capaz de avaliar a segurança e a eficácia dos produtos sob revisão. Também é essencial que o representante tenha uma afiliação e/ou participação ativa em organizações representativas de pacientes (ORP) ou comunitárias e declarar os potenciais conflitos de interesses. O representante do paciente tem como missão refletir a perspectiva do consumidor em questões e ações perante o comitê consultivo; servir como elo entre a comissão e os consumidores, e entre aquela e as ORP interessadas; bem como facilitar o diálogo com os comitês consultivos sobre questões científicas que afetam os consumidores (11) (ver Figura 1). 
Figura 1. Critérios para se tornar um representante de pacientes da FDA (FDA Patient Representative Program ${ }^{\mathrm{sm}}$ )


Fonte: Adaptado da FDA (11).

A FDA e a EMA também criaram um grupo de trabalho chamado FDA/EMA Patient Engagement Cluster, permitindo que essas agências compartilhem as melhores práticas que possibilitem o envolvimento dos pacientes ao longo do ciclo dos processos regulatórios dos medicamentos. Dentre os desafios que são discutidos pelo grupo estão a busca por uma comunicação efetiva entre todos os envolvidos; treinamento para os representantes; e estratégias para coletar dados sobre o impacto da participação dos pacientes (11).

Podemos agregar diferentes argumentos para discutir a importância e os desafios da participação social na regulação e vigilância de medicamentos. Inevitavelmente, a viabilidade dessa passa pelo letramento ou alfabetização científica do paciente e pela capacidade de comunicação dos profissionais da saúde e técnicos das agências reguladoras, além do aprimoramento de mecanismos de participação social.

Um estudo realizado pelo Instituto Abramundo sobre letramento científico realizado em 2014 em nove das principais regiões metropolitanas do Brasil (com 2.002 entrevistados), classificou $48 \%$ dos entrevistados com letramento científico rudimentar (elementar), que se refere à falta de domínio de conhecimentos e habilidades para resolver problemas ou interpretar informações científicas. Apenas $5 \%$ foram classificados com letramento científico proficiente, enquanto $31 \%$ foram classificados com letramento científico básico e $16 \%$ classificados com letramento não-científico. A título comparativo, outra pesquisa realizada no Canadá em 2012 estimou que 42\% dos adultos possuíam um nível de letramento científico adequado $(17,18)$.

Por outro lado, o uso de terminologia técnica ou aspectos linguísticos não deveriam ser barreiras para a participação social em instituições públicas. Para que a participação 
social em saúde seja mais efetiva, é preciso evitar que as instituições marginalizem as contribuições sociais nas discussões mais técnicas, relegando a fala do paciente a um saber leigo. Logo, se existem dificuldades de comunicação dos profissionais com os leigos, "a crise de interpretação é nossa" (19).

Diante das dificuldades apresentadas, a contribuição das ORP seria uma alternativa para a inclusão dos pacientes na vigilância de medicamentos.

Algumas ORP estão se profissionalizando e podem possuir diferentes quadros técnicos com capacidade de atuar em temas técnicos complexos e experiência na comunicação para o paciente, inclusive com capacidade de auxiliar o governo em atividades do legislativo e executivo. Como exemplo de capacidade técnica, comunicação e colaboração governamental, podemos citar a Associação Brasileira de Linfoma e Leucemia (Abrale), uma organização social centrada no paciente que lançou, em 2015, o Observatório da Oncologia, uma plataforma online de monitoramento de dados abertos e compartilhamento de informações na área de oncologia no Brasil e que vem contribuindo com o diagnóstico, tratamento precoce e melhores investimentos em saúde (20).

As organizações atuam intensa e eficazmente no apoio a grupos de pacientes e no enfrentamento das dificuldades relacionadas a doenças específicas (21). As ORP também estão ampliando o seu espectro de ação, cobrindo uma grande variedade de demandas em saúde, por meio de advocacy, participação em órgãos públicos, atividades de comunicação e campanhas de conscientização (22), e poderiam auxiliar sistematicamente no sistema de farmacovigilância, em atividades como a rede sentinela, notificação de EAM, avaliação de tecnologias em saúde, comunicação em saúde, dentre outros.

As organizações representativas de pacientes emergem em todo o mundo como novos espaços de participação social na área da saúde, utilizando intervenções inovadoras na promoção de direitos de pessoas com necessidades em saúde, promovendo e organizando plataformas de coalizão nacional e transnacional, bem como atuando em áreas antes reservadas a pesquisadores e profissionais da saúde. As ORP podem apresentar um forte envolvimento na pesquisa de doenças raras sem tratamento disponível, em questões relacionadas ao acesso a medicamentos ou à desmedicalização, bem como no reconhecimento de direitos humanos (23). Por outro lado, muitas apresentam dificuldades 
em termos de capacitação organizacional para a atuação eficiente nas suas diversas frentes de atuação.

No Brasil, a Anvisa vem realizando alguns esforços para aproximar a população, como o projeto Educação em Vigilância Sanitária (Educanvisa), que busca capacitar professores da rede pública de ensino em assuntos relativos à vigilância sanitária para uso na comunidade escolar. A ideia central do Educanvisa é alertar sobre os riscos associados ao uso inadequado de produtos sujeitos ao controle sanitário. Entre 2006 e 2017, o projeto atendeu, diretamente, mais de 200 mil alunos de 1.456 escolas públicas brasileiras, além de promover a formação de mais de sete mil professores (24).

No contexto brasileiro, cabe ressaltar que a Anvisa também utiliza diversos mecanismos de participação social, transparência e accountability na sua gestão, mas reconhece as dificuldades na efetivação desses mecanismos. Atualmente, o órgão disponibiliza os seguintes mecanismos de participação social: ouvidoria, audiências públicas, consultas públicas, editais de chamamento, diálogos setoriais, tomadas públicas de subsídios, além dos conselhos e órgãos colegiados (24).

Entretanto, segundo Coutinho e Mizara (2), o único mecanismo institucional de participação social considerado com alguma efetividade, no âmbito da Anvisa, foi o Conselho consultivo, que possibilita a participação de representantes do setor público, privado, comunidade científica e da sociedade civil organizada. Os demais mecanismos, por sua vez, possuem graves problemas, destacadamente quanto à transparência e à paridade da participação social, com uma sub-representação dos consumidores/pacientes e a predominância do setor produtivo.

Sublinha-se que a Política Nacional de Participação Social reconhece a necessidade de fortalecer os mecanismos e as instâncias democráticas de diálogo e a atuação conjunta entre o governo e a sociedade civil em todas as fases das políticas públicas e no aprimoramento da gestão pública (25).

\section{Conclusão}

A participação social em saúde é resultado de um longo processo de lutas e engajamento social no Brasil, que está se estruturando no SUS por meio da Constituição e dos principais marcos legais do sistema (Lei 8.080 e 8.142, de 1990, e Normas Operacionais 
Básicas 1/91, 1/92 e 1/96), bem como por meio de mecanismos de participação direta como conselhos e conferências. Apesar desses avanços institucionais, a participação social em saúde no Brasil ainda se encontra muito aquém das suas possibilidades (26), como é o caso da participação de pacientes na regulação e vigilância de medicamentos, que já se verifica na Europa ou Estados Unidos, mas ainda incipiente no contexto brasileiro.

Existem iniciativas consolidadas e resultados que sustentam os benefícios em engajar os pacientes na vigilância de medicamentos. Notadamente, algumas organizações representativas de pacientes podem contribuir mais efetivamente nas diversas fases de desenvolvimento dos medicamentos e comunicação entre as agências reguladoras e os pacientes. Por outro lado, é fundamental que as agências busquem aprimorar os mecanismos de participação e a utilização de métodos que possibilitem coletar as contribuições sociais e a sistematização das experiências dos pacientes. Certamente, um dos grandes desafios será a mudança de perspectiva, de um cidadão passivo na relação de consumo para um cidadão que, a partir das suas experiências como paciente, pode contribuir ativamente com a segurança dos medicamentos no mercado, o desenvolvimento de fármacos e, assim, com a qualidade de todo o sistema de saúde.

\section{Referências}

1. Oliveira AMC, Dallari SG. Participação social no planejamento das ações e serviços de vigilância sanitária: uma análise crítica. Revista Vigilância Sanitária em Debate. 2014, 2(2): 9-16. Disponível em:

https://www.researchgate.net/publication/272738799_Participacao_social_no_planejament o_das_acoes_e_servicos_de_vigilancia_sanitaria_uma_analise_critica. [Ācesso em 20.mar.2018].

2. Coutinho DR, Miziara N. Participação social, transparência e accountability na regulação sanitária da Anvisa. In: Aith F, Dallari SG, organizadores. Regulação de medicamentos no mundo globalizado. São Paulo: Centro de Estudos e Pesquisas de Direito Sanitário (CEPEDISA); 2014. p. 305-335. Disponível em: http://napdisa.prp.usp.br/wpcontent/uploads/2016/10/drugs_regulation_online_sep14.pdf. [Acesso em 2.nov.2017].

3. Barnes M, Coelho VS. Social participation in health in Brazil and England: inclusion, representation and authority. Health Expectations. (8 set 2009), 12 (3):226-36. doi: 10.1111/j.1369-7625.2009.00563.x.

4. Durán PRF, Gerschman S. Desafios da participação social nos conselhos de saúde. Saúde e Sociedade. (2014), 23 (3):884-896. DOI: 10.1590/S0104-12902014000300012 
5. Brasil. Senado Federal. Decreto Legislativo no 273, de 2014. Susta a Resolução - RDC ํㅡ 52, de 6 de outubro de 2011, da Agência Nacional de Vigilância Sanitária - ANVISA, que dispõe sobre a proibição do uso das substâncias anfepramona, femproporex e mazindol, seus sais e isômeros, bem como intermediários e medidas de controle da prescrição e dispensação de medicamentos que contenham a substância sibutramina, seus sais e isômeros, bem como intermediários. Disponível em:

http://www2.camara.leg.br/legin/fed/decleg/2014/decretolegislativo-273-4-setembro-2014779343-publicacaooriginal-144957-pl.html. [Acesso em 2.set.2016].

6. Brasil. Ministério da Saúde Agência Nacional de Vigilância Sanitária. Resolução da Diretoria Colegiada - RDC № 50, de 25 de setembro de 2014. Dispõe sobre as medidas de controle de comercialização, prescrição e dispensação de medicamentos que contenham as substâncias anfepramona, femproporex, mazindol e sibutramina, seus sais e isômeros, bem como intermediários e dá outras providências. Disponível em:

http://portal.anvisa.gov.br/documents/33880/2568070/reprdc0050_25_09_2014.pdf/d04dec 76-4dbb-4d04-a721-50bd191a1a9b?version=1.0. [Acesso em 3.jul.2018].

7. Pinto JM. Bulas de medicamentos comercializados no Brasil enquanto fontes de informação: em foco a qualidade da informação nelas contidas após a Resolução - RDC no 47/2009 da ANVISA [Dissertação]. Minas Gerais: Programa de Pós-Graduação em Ciência da Informação, Escola de Ciência da Informação da Universidade Federal de Minas Gerais. 2013.

8. Volpato LF, Martins LC, Mialhe FL. Bulas de medicamentos e profissionais de saúde: ajudam ou complicam a compreensão dos usuários? Revista de Ciências Farmacêuticas Básica e Aplicada. 2009, 30(3):309-314. Disponível em: http://servbib.fcfar.unesp.br/seer/index.php/Cien_Farm/article/viewFile/623/887. [Acesso em 3.jul.2018].

9. Eggertson L. Drug approval system questioned in US and Canada. Canadian Medical Association Journal. (1 fev 2005), 172(3):317-318. DOI: 10.1503/cmaj.045312.

10. European Medicines Agency. [Relatório Técnico]. Stakeholder engagement report 2017: patients, consumers, healthcare professionals and their organisations. 2017. Disponível em: https://www.ema.europa.eu/documents/report/stakeholder-engagementreport-2017_en.pdf. [Acesso em 3.jul.2018].

11. US Food and Drug Administration. Learn About FDA Patient Engagement. Disponível em: https://www.fda.gov/ForPatients/PatientEngagement/default.htm\#peac. [Acesso em 3.jul.2018].

12. Suvarna V. Phase IV of Drug Development. Perspectives in Clinical Research. 2010, Apr-Jun; 1(2): 57-60. Disponível em: https://www.ncbi.nlm.nih.gov/pmc/articles/PMC3148611/. [Acesso em 20.mar.2018]. 
13. Carpenter D, Zucker EJ, Avorn J. Drug-review deadlines and safety problems. The New England Journal of Medicine. (27 mar 2008), 358(13):1354-1361. DOI:

10.1056/NEJMsa0706341

14. Organização Mundial da Saúde. Departamento de Medicamentos Essenciais e outros Medicamentos. A importância da Farmacovigilância. Brasília: Organização Pan-Americana da Saúde, 2005. Disponível em:

http://bvsms.saude.gov.br/bvs/publicacoes/importancia.pdf. [Acesso em 20.mar.2018].

15. Herxheimer A, Crombag R, Alves TL. Direct Patient Reporting of Adverse Drug

Reactions: A Fifteen-Country Survey \& Literature review. Health Action International (HAl) Europe, 2010. Disponível em:

https://consumers.cochrane.org/sites/consumers.cochrane.org/files/public/uploads/10\%20M ay\%202010\%20Report\%20Direct\%20Patient\%20Reporting\%20of\%20ADRs.pdf. [Acesso em 20.mar.2018].

16. van Hunsel F, Härmark L, Pal S, Olsson S, van Grootheest K. Experiences with adverse drug reaction reporting by patients: an 11-country survey. Drug Saf. (1.jan.2012), 35(1):4560. DOI: $10.2165 / 11594320-000000000-00000$.

17. Instituto Abramundo [Relatório Técnico]. Letramento Científico: Um Indicador para o Brasil, versão preliminar. 2014. Disponível em:

http://www.observatoriodopne.org.br/uploads/reference/file/580/documento-referencia.pdf. [Acesso em 10.out.2018].

18. Instituto Abramundo [Relatório Técnico]. Indicador de Letramento Científico: Relatório técnico da edição 2014. 2014. Disponível em: http://www.ibope.com.br/ptbr/noticias/Documents/Relatorio_Final_ILC_JUL2014.pdf. [Acesso em 10.out.2018].

19. Valla VV. Controle Social ou Controle Público. In: De Seta MH, Pepe VLE, Oliveira GO, organizadores. Gestão e vigilância sanitária: modos atuais do pensar e fazer. Rio de Janeiro: Ed. Fiocruz, 2006.

20. Associação Brasileira de Linfoma e Leucemia. Observatório de Oncologia. Disponível em: https://observatoriodeoncologia.com.br/\#midia. [Acesso em 19.ago.2018].

21. Moretti FA, Zucchi P. Caracterização dos grupos de apoio e associações de pacientes portadores de doença reumatológica no Brasil. Rev. Bras. Reumatol, 2010; 50 ( 5 ): 516528.

22. Mosconi P, Colombo C, Satolli R, Liberati A. PartecipaSalute, an Italian project to involve lay people, patients' associations and scientific-medical representatives in the health debate. Health Expectations. (22.maio.2007), 10(2):194-204. Erratum in: Health Expect. 2007 Sep;10(3):305. DOI: 10.1111/j.1369-7625.2007.00444.x

23. Nunes JA, Matias M. As organizações de pacientes como atores emergentes no espaço da saúde: o caso de Portugal. Revista Eletrônica de Comunicação, Informação e 
Inovação em Saúde. (jan.-jun.2007), 1(1): 107-110. DOI: http://dx.doi.org/10.3395/reciis.v1i1.894

24. Brasil. Ministério da Saúde. Agência Nacional de Vigilância Sanitária. Educanvisa: Educação em Vigilância Sanitária. Encontro Educação e Saúde lança livro sobre Educanvisa. Disponível em: http://portal.anvisa.gov.br/educanvisa. [Acesso em 20.set.2018].

25. Brasil. Presidência da República, Casa Civil, Subchefia para Assuntos Jurídicos. Decreto oㅡ 8.243, de 23 de maio de 2014. Institui a Política Nacional de Participação Social - PNPS e o Sistema Nacional de Participação Social - SNPS, e dá outras providências. Disponível em: http://www.planalto.gov.br/ccivil_03/_ato20112014/2014/decreto/d8243.htm. [Acesso em 2.set.2016].

26. Vázquez ML, Ferreira Silva MR, Gonzalez ESC, Diniz AS, Pereira APC, Veras ICL, Arruda IKG. Nível de informação da população e utilização dos mecanismos institucionais de participação social em saúde em dois municípios do Nordeste do Brasil. Ciência e saúde coletiva. (set.dez.2005), 10(Suppl):141-155. DOI: http://dx.doi.org/10.1590/S141381232005000500017

Soares JR, Oliveira VE. A participação social na vigilância de medicamentos. Cadernos Ibero-Americanos de Direito Sanitário. 2019 abr./jun.; 8(2): 31-43. 